\title{
Survey on practice, perception and knowledge of doctors on antibiotic use and resistance in Penang government hospitals, Malaysia
}

\author{
Lim Phin Phin ${ }^{1}$, Teoh Chee Jia ${ }^{2}$, Chua Cheah Chin 3 , Sherene Tan Su Ann4, Ch'ng Pao Pao \\ Liew Ai Ch'i ${ }^{5}$, Celine Choong Yen Ching ${ }^{6}$, Foong Weng Siang7 ${ }^{7}$, Lee Yi Hui ${ }^{1}$, Lim Eu Joon ${ }^{1}$, \\ Hoo Shi Min ${ }^{1}$, Wong Peng Shyan ${ }^{8}$
}

${ }^{1}$ Department of Pharmacy, Hospital Kepala Batas, Penang, Ministry of Health Malaysia.

2 Department of Pharmacy, Hospital Seberang Jaya, Penang, Ministry of Health Malaysia.

${ }^{3}$ Department of Pharmacy, Klinik Kesihatan Kubang Semang, Penang, Ministry of Health Malaysia.

${ }^{4}$ Department of Pharmacy, Hospital Pulau Pinang, Penang, Ministry of Health Malaysia.

${ }^{5}$ Clinical Research Centre, Hospital Seberang Jaya, Penang, Ministry of Health Malaysia.

${ }^{6}$ Department of Pharmacy, Hospital Bukit Mertajam, Penang, Ministry of Health Malaysia.

7 Department of Pharmacy, Hospital Balik Pulau, Penang, Ministry of Health Malaysia.

${ }^{8}$ Infectious Diseases Unit, Department of Medicine, Hospital Pulau Pinang, Penang,

Ministry of Health Malaysia.

DOI: 10.3396/ijic.v16i3.020.20

\begin{abstract}
Inappropriate prescribing of antibiotics is one of the factors that leads to the development of antibiotic resistance. Therefore, understanding doctors' practice, perception and knowledge is vital in targeting strategies to prevent antibiotic resistance. The aim of the study is to determine the practice, perception and knowledge of doctors on antibiotic use and resistance in Penang government hospitals. This is a cross sectional survey carried out in six Penang government hospitals from 9-20 January 2017. A face-and-content validated questionnaire adapted from Abbo et al., was distributed to house officers (HO), medical officers (MO) and specialists/consultants (SC) and collected back within five working days. A total of 243 questionnaires were completed with a response rate of $60.45 \%$. Majority of our respondents (91.6\%) considered whether patient is critically ill and/or immunocompromised when selecting an antibiotic. Regardless of their position, doctors agreed that antibiotics are overused and antibiotic resistance is a significant problem nationally. However, MO tended to perceive antibiotic management programmes as an obstacle to good patient care. The mean knowledge score among respondents was $5.88 \pm 1.92$ out of 10 . Adjusted analysis showed that $\mathrm{SC}$ have significantly higher mean knowledge score compared to $\mathrm{HO}$ and $\mathrm{MO}(\mathrm{p}<0.001)$. Current antibiotic management programmes need to be continued to ensure judicious antibiotic use. However, a more targeted antimicrobial education strategy based on the findings of this study should be carried out to improve the practice, perception and knowledge of doctors on antibiotic use and resistance.
\end{abstract}

Keywords: antibiotic resistance; knowledge, attitudes, practice; physicians; Malaysia

Corresponding author:

Lim Phin Phin

Department of Pharmacy, Hospital Kepala Batas, Jalan Bertam 2, 13200, Penang, Malaysia.

Email: icephin@gmail.com 


\section{Introduction}

Antibiotics are among the most frequently prescribed medications worldwide. ${ }^{1}$ They are used in multiple disciplines to treat a myriad of conditions involving infections. ${ }^{2}$ Nevertheless, widespread usage of antibiotics has given rise to antibiotic resistance. With limited new antibiotics in the pipeline, antibiotic resistance needs to be addressed urgently.

Antibiotic usage in Malaysian government hospitals is monitored using defined daily dose (DDD) based on World Health Organisation (WHO) standard. The antibiotics commonly used in hospitals were cephalosporins, fluoroquinolones, carbapenems, glycopeptides and penicillin/beta-lactamase inhibitors. Frequent use of these antibiotics has contributed to the increased resistance towards these agents. According to the National Surveillance of Antibiotic Resistance (NSAR), antibiotic resistance in Malaysia has shown an increasing trend. Meticillinresistant Staphylococcus aureus (MRSA) rate has increased from $18 \%$ (2016) to $19.8 \%$ (2017). For Escherichia coli, resistance to cefuroxime has increased from $24.8 \%$ (2016) to $26.7 \%$ (2017). The meropenemresistant Klebsiella pneumoniae (CRKP) has also shown a tremendous rise from $2.6 \%$ (2016) to $4.7 \%$ (2017). ${ }^{3}$ All these are alarming signs indicating that judicious use of antibiotics need to be prioritised. Apart from the excessive use of antibiotics, another factor that leads to antibiotic resistance includes insufficient antibiotic control policies. ${ }^{4}$ Antibiotic stewardship programmes (ASP) have been introduced in Malaysian government hospitals since $2014 .{ }^{5}$ In Penang governmenthospitals, the main antibiotic management programme adopted is formulary restriction in which the usage of selected antibiotics in each hospital is controlled by their specialists/consultants (SC). For these antibiotics, either pre-approval by SC is required before initiation, or temporary approval is given where the antibiotic can be started by medical officers (MO) or house officers $(\mathrm{HO})$ but would need approval by SC for continued use. $\mathrm{HO}$ refers to doctors who are still in their housemanship, whereas MO refers to the doctors who have passed their housemanship and working in the hospital. Other than that, certain selected antibiotics will need to be reviewed by SC 72 hours after initiation and justification is needed for continuation.
Doctors are key persons in deciding the choice of antibiotics, dose and duration of treatment. A previous study identified that insufficient knowledge on guidelines and procedures have resulted in unsatisfactory prescribing patterns, and physicians' perceptions on antibiotic resistance were often diverse and contradictory to one another. ${ }^{6}$ Such varying levels of knowledge and opinions among doctors may potentially hinder the success of ASP. Therefore, it is imperative to understand doctors' practice, perception and knowledge in order to develop effective strategies to prevent antibiotic resistance.

\section{Methods}

This is a cross sectional survey carried out in six Penang government hospitals from 9-20 January 2017. The 56-item questionnaire was adapted from a study conducted by Abbo et al. and was face and content validated. ${ }^{7}$ The author has given consent for the use of the questionnaire in this study. It consisted of four sections including demographic data, prescribing practices, perception of respondents towards antibiotics use and resistance and lastly, knowledge of the respondents.

Information on age, gender, the number of years in practice, whether the respondent was graduated in local or foreign university, position in hospital $(\mathrm{HO}$, $\mathrm{MO}$ or $\mathrm{SC}$ ) and area of practice were included in the demographic section. For questions on the practices, a 5-point Likert scale with options ranged from "never" to "always" was used. For the perceptions section, options ranged from "strongly disagree" to "strongly agree" were used. For knowledge section, 10 multiple-choice questions were included and there was only one correct answer for each question. One point was given for each correct answer therefore the maximum knowledge score for a respondent was 10 . The questionnaires were distributed to all doctors available during the data collection period by designated data collectors and were collected back by the same person within five working days.

All data analysis was performed with Statistical Package of Social Sciences (SPSS) version 16.0 (IBM, Armonk, NY). ANOVA test or Kruskal-Wallis test was performed to compare practice, perception or 
knowledge score with demographic characteristics. If $p$-value $<0.05$, post-hoc analysis was carried out to confirm where the differences occurred between groups.

All doctors practicing in general medicine, critical care, surgery, orthopaedics, neurology, nephrology, endocrinology, cardiology, infectious disease (ID) and respiratory departments who were on duty during the study period were included. Visiting doctors, locum doctors, doctors on administrative duty or involved in non-clinical work and doctors who refused to participate in the survey were excluded.

This study has been granted ethical approval from Medical Research and Ethics Committee Ministry of Health Malaysia.

\section{Results}

\section{Demography}

Out of the 402 survey forms distributed, 243 (60.45\%) were completed. HO made up $25.2 \%$ of the respondents, $\mathrm{MO}$ being a majority at $57 \%$ while $17.8 \%$ were SC. The total number of doctors was 714 , made up of $\mathrm{HO}(39.2 \%), \mathrm{MO}(44 \%)$ and SC (16.8\%). The median age of the respondents was 29 years old, and $51.7 \%$ of the respondents were male. Most of the respondents have been practising for 1-5 years (52.9\%), followed by less than a year (19.8\%), 6-10 years (13.2\%), $11-15$ years (6.6\%), $16-20$ years $(5.4 \%)$, and more than 20 years $(2.1 \%)$ respectively. More than half of the respondents $(61.6 \%)$ graduated from local universities while the other $38.4 \%$ graduated from foreign universities.

\section{Practice}

The majority of our respondents spent their clinical time in inpatient settings (64\%). Thirty percent of them spent approximately equal time between outpatient and inpatient. Eighty one percent of the respondents usually prescribe antibiotics for inpatients, $2 \%$ for outpatients and $17 \%$ for both.

Almost all (91.6\%) of our respondents often or always considered whether the patient is critically ill and/or immunocompromised when selecting an antibiotic. Around half of our respondents often or always considered the risk of missing an infection and unexplained fever or leucocytosis even if the culture were negative and only $25 \%$ of the respondents took into consideration cost saving for the hospital when selecting an antibiotic. Thirty percent of the respondents were often or always influenced by reassurance from colleagues when prescribing an antibiotic even if it is not correctly prescribed. Further analysis showed that cost savings significantly influenced the decision of $\mathrm{MO}$ and SC compared to $\mathrm{HO}(\mathrm{p}=0.012)$, criteria of patient being critically ill and/or immunocompromised strongly influenced the decision of SC compared to $\mathrm{HO}$ and $\mathrm{MO}$ ( $\mathrm{p}=$ 0.021), while reassurance when using an incorrectly prescribed antibiotic significantly influenced the decision of $\mathrm{HO}$ and $\mathrm{MO}$ more than $\mathrm{SC}(\mathrm{p}<0.001)$. The result is displayed in Table I.

The most popular source of information on antibiotics among our respondents was ward rotations (98.3\%), followed by grand rounds, continuous medical education (CME) and medical journals with equal frequency of $97.5 \%$ each. Ninety seven percent of the respondents chose to refer to the ID colleague or pharmaceutical representative for information. Other sources of information include lecture sponsored by pharmaceutical company (91.1\%), ASP (85.5\%), Infectious Diseases Society of America (IDSA) guidelines (84.9\%), the Sanford Guide (81.9\%) and the internet (27.2\%).

\section{Perception}

Perceptions of doctors regarding antibiotics use and resistance are summarized in Table II. All respondents unequivocally agreed that appropriate use of antibiotics will reduce problems with antibioticresistant organisms and strong knowledge of antibiotics is imperative in one's medical career. SC and $\mathrm{MO}$ were significantly more confident compared to $\mathrm{HO}(p<0.001)$ in using antibiotics optimally. As compared to SC, MO tended to perceive antibiotic management programmes as an obstacle to good patient care $(p=0.001)$. However, HO perceived that they need more education on antibiotics as compared to $M O(p=0.011)$ and both $\mathrm{HO}$ and MO were less likely to use restricted antibiotics if a specialist's approval was needed. 


\section{Knowledge}

The mean knowledge score of the respondents was $5.88 \pm 1.92$ out of 10 . Percentage of correct answers by respondents for each question is summarized in Table III. The question related to the management of MRSA bacteraemia obtained the highest percentage of correct answers at $92.2 \%$, while question on the knowledge of antibiotic use in surgical prophylaxis garnered the lowest (21\%).

As shown in Table IV, there was no significant difference in mean scores between genders, places of graduation and primary work areas, although respondents from the endocrinology unit obtained the highest mean score $(7.50 \pm 2.12)$ while those from the surgical department scored the lowest $(4.33 \pm 2.77)$. Unadjusted analysis found significant difference in mean scores between respondents with less years of practice compared to respondents with more years of practice. The mean scores between respondents of different positions also showed significant difference (HO, 5.18 \pm 1.53 ; MO, 5.67 $\pm 1.95 ; \mathrm{SC}, 7.57 \pm 1.31$; $\mathrm{p}<0.001)$. In addition, when the mean scores between respondents of different positions were subjected to general linear model analysis (Table V), post-hoc
Bonferroni's procedure demonstrated significant difference between $\mathrm{HO}$ and $\mathrm{SC}(\mathrm{p}<0.001)$ and between $\mathrm{MO}$ and $\mathrm{SC}(\mathrm{p}<0.001)$.

\section{Discussion}

Practice

Our study shows that the major factors influencing antibiotic prescribing were the patient's clinical condition, presence of unexplained fever or leucocytosis in the absence of a positive culture, and the risk of missing an infection. This is similar to the findings by Venugopalan et al..$^{8}$ This can be explained by the higher risk of death if antibiotic is not commenced early in patients with serious infections. ${ }^{9}$

Cost saving for the hospital was not a major factor that influenced the antibiotic selection for most of our respondents, concurring with the findings reported by Venugopalan et al. and Baadani et al.,10 Our study showed that $\mathrm{HO}$ was the least influenced by cost compared to $\mathrm{MO}$ and SC. In Malaysia, the cost of healthcare in government hospitals is mostly funded by the government. Budget allocation for Ministry of Health has been increased by $7.8 \%$ for year 2019. ${ }^{11}$ The Malaysian Statistics on Medicines (MSOM)

Table I. Influence on decision to select an antibiotic by position

Influence on decision to select an antibiotic by position

\begin{tabular}{|c|c|c|c|c|}
\hline 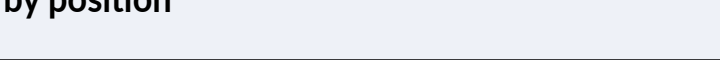 & SC & MO & $\mathrm{HO}$ & \\
\hline Cost savings for the hospital & $3.0(1.00)$ & $3.0(2.00)$ & $3.0(1.00)$ & $0.012^{\mathrm{a}}$ \\
\hline Risk of missing of an infection & $4.0(2.00)$ & $4.0(1.00)$ & $3.5(1.00)$ & 0.424 \\
\hline $\begin{array}{l}\text { The patient is critically ill and/or } \\
\text { immunocompromised }\end{array}$ & $5.0(0.00)$ & $5.0(1.00)$ & $5.0(1.00)$ & $0.021^{b}$ \\
\hline $\begin{array}{l}\text { Reassurance when using an antibiotic even if it } \\
\text { might be the wrong one }\end{array}$ & $2.0(1.00)$ & $3.0(2.00)$ & $3.0(1.00)$ & $<0.001^{c}$ \\
\hline $\begin{array}{l}\text { Unexplained fever or leukocytosis even if } \\
\text { cultures are negative }\end{array}$ & $3.0(1.00)$ & $4.0(1.00)$ & $4.0(1.00)$ & 0.597 \\
\hline
\end{tabular}

Note: *Kruskal Wallis

apost hoc shown significant difference between $\mathrm{HO}$ vs $\mathrm{MO}$ and $\mathrm{HO}$ vs $\mathrm{SC}$ bpost hoc shown significant difference between SC vs $\mathrm{HO}$ and SC vs MO cpost hoc shown significant difference between SC vs $\mathrm{HO}$ and SC vs MO 
Table II. Agreement of doctors with statements regarding perceptions about antimicrobial use and resistance

\section{Perceptions of doctors regarding antibiotics use and resistance}

\begin{tabular}{|c|c|c|c|c|}
\hline & SC & MO & $\mathrm{HO}$ & p-value* \\
\hline Antibiotics are overused nationally & $4.0(1.00)$ & $4.0(1.00)$ & $4.0(1.00)$ & 0.547 \\
\hline Antibiotic resistance is a significant problem nationally & $4.0(1.00)$ & $4.0(1.00)$ & $4.0(1.00)$ & 0.254 \\
\hline $\begin{array}{l}\text { Better use of antibiotics will reduce problems with } \\
\text { antibiotic-resistant organisms }\end{array}$ & $5.0(1.00)$ & $5.0(1.00)$ & $5.0(1.00)$ & 0.149 \\
\hline $\begin{array}{l}\text { Strong knowledge of antibiotics is important in my medical } \\
\text { career }\end{array}$ & $5.0(1.00)$ & $5.0(1.00)$ & $5.0(1.00)$ & 0.519 \\
\hline I am confident that I use antibiotics optimally & $4.0(0.25)$ & $4.0(1.00)$ & $3.0(1.00)$ & $<0.001^{\mathrm{a}}$ \\
\hline $\begin{array}{l}\text { Antibiotic utilization/management programmes are an } \\
\text { obstacle to good patient care }\end{array}$ & $2.0(1.00)$ & $3.0(1.00)$ & $3.0(2.00)$ & $0.001^{b}$ \\
\hline I would like more feedback on my antibiotic selection & $4.0(2.00)$ & $4.0(0.00)$ & $4.0(0.75)$ & 0.521 \\
\hline I would like more education on antibiotics & $4.0(1.00)$ & $4.0(1.00)$ & $5.0(1.00)$ & $0.011^{c}$ \\
\hline $\begin{array}{l}\text { I am less likely to use restricted antibiotics if a specialist's } \\
\text { approval is required }\end{array}$ & $3.0(2.00)$ & $4.0(1.00)$ & $3.0(1.00)$ & $0.004^{d}$ \\
\hline $\begin{array}{l}\text { Institutionally developed guidelines for antibiotic treatment } \\
\text { would be more useful to me than national guidelines }\end{array}$ & $3.5(1.00)$ & $4.0(1.00)$ & $4.0(1.00)$ & 0.345 \\
\hline $\begin{array}{l}\text { I am concerned about antimicrobial resistance in the society } \\
\text { when I prescribe antibiotics }\end{array}$ & $4.0(1.00)$ & $4.0(0.00)$ & $4.0(1.00)$ & 0.338 \\
\hline $\begin{array}{l}\text { I am concerned about antimicrobial resistance in my } \\
\text { hospital when I prescribe antibiotics }\end{array}$ & $4.0(1.00)$ & $4.0(0.00)$ & $4.0(1.00)$ & 0.438 \\
\hline $\begin{array}{l}\text { Prescribing broad-spectrum antibiotics when equally } \\
\text { effective narrower ones are available increases antimicrobial } \\
\text { resistance }\end{array}$ & $4.5(1.00)$ & $4.0(1.00)$ & $4.0(1.75)$ & $0.014^{d}$ \\
\hline $\begin{array}{l}\text { Poor infection control practices by healthcare professionals } \\
\text { cause spread of antimicrobial resistance }\end{array}$ & $4.5(1.00)$ & $4.0(1.00)$ & $4.0(1.00)$ & 0.072 \\
\hline $\begin{array}{l}\text { Inappropriate use of antibiotics causes antimicrobial } \\
\text { resistance }\end{array}$ & $5.0(1.00)$ & $4.0(1.00)$ & $5.0(1.00)$ & $0.015^{b}$ \\
\hline Inappropriate use of antibiotics can harm patients & $4.5(1.00)$ & $4.0(1.00)$ & $4.0(1.00)$ & 0.139 \\
\hline Inappropriate use of antibiotics is professionally unethical & $4.0(2.00)$ & $4.0(1.00)$ & $4.0(2.00)$ & 0.812 \\
\hline $\begin{array}{l}\text { A proper antibiotic stewardship program is necessary in my } \\
\text { hospital }\end{array}$ & $4.0(1.00)$ & $4.0(1.00)$ & $4.0(1.00)$ & 0.286 \\
\hline $\begin{array}{l}\text { Intravenous antibiotics should be changed to oral } \\
\text { immediately when patient can take orally / improves } \\
\text { clinically }\end{array}$ & $4.0(2.00)$ & $4.0(1.00)$ & $4.0(1.00)$ & 0.780 \\
\hline
\end{tabular}

SC, Specialist/ Consultant; MO, Medical Officer; HO, House Officer.

Note: *Kruskal Wallis

apost hoc shown significant difference among all sub groups

${ }^{b}$ post hoc shown significant difference between MO versus SC

cpost hoc shown significant difference between $\mathrm{HO}$ versus $\mathrm{MO}$

${ }^{d}$ post hoc shown significant difference between $\mathrm{HO}$ versus $\mathrm{SC}$ and $\mathrm{MO}$ versus $\mathrm{SC}$ 
project 2011-2014 reported that systemic antibiotics ranked the highest among all therapeutic drugs with increasing expenditure from RM (Malaysian ringgit) 381 million (2011) to RM 422 million (2014).12 This highlights the need to raise awareness among our respondents, especially $\mathrm{HO}$, on the cost of antibiotics in order to inculcate the habit of cost-efficient prescribing.

Our study found that $\mathrm{HO}$ and $\mathrm{MO}$ were significantly more likely to be reassured by colleagues when using an antibiotic, even though it was not appropriately prescribed. This reflects the respondents' lack of knowledge of antibiotics to make a confident decision. An effective way to improve this is to provide more training and exposure to adequately equip prescribers with the necessary knowledge and confidence.

Ward rotations, grand rounds, CME, medical journals, ID colleagues and pharmaceutical representatives were among the main sources of information for continuing education on antibiotics among our respondents. A study by Sunenshine et al. showed $89 \%$ of the practitioners agreed that ID consultants need to be involved directly in the approval of use for selected antibiotics. ${ }^{13}$ Another study by AlTawfik from Saudi Arabia reported that $58.7 \%$ of ID consultation cases had resulted in the change of antimicrobial therapy and discontinuation of inappropriate antimicrobials. ${ }^{14}$ On the other hand, a study by Honda et al. showed a $56 \%$ reduction in 28 day mortality after ID consultation in patients with Staphylococcus aureus bacteraemia. ${ }^{15}$ These showed that ID consultation should be routinely sought to ensure judicious use of antibiotics and to improve patient outcome.

Our respondents also considered pharmaceutical company-sponsored lectures and pharmaceutical representatives as the main sources of information, similar to findings from some studies. ${ }^{16,17}$ However, there were other studies where these were rated as less useful. ${ }^{7,8,18}$ Respondents should be cautious for risk of bias when acquiring information from pharmaceutical companies. Avorn et al. found that although most physicians perceived themselves to have little influence by the drug advertisements, their degree of believe were revealed to be the opposite. ${ }^{19}$

The IDSA guidelines and Sanford Guide are the main references utilized worldwide in aiding antimicrobial prescribing. However, their rank in our study was lower than pharmaceutical company. This highlights the need to further promote the use of guidelines to ensure evidence-based prescribing.

Table III. Percentage of correct answers by respondents.

Questions Correct answers by respondents (\%)

\begin{tabular}{lr}
\hline Management of MRSA bacteremia & 92.2 \\
\hline Management of ESBL positive E. coli bacteremia & 74.5 \\
\hline Risk of antibiotics to develop Clostridium difficile colitis & 46.9 \\
\hline Appropriate use of IV vancomycin & 42.4 \\
\hline Appropriate antibiotic use in hospitalised patients & 73.5 \\
\hline Antibiotic use in surgical prophylaxis & 21.0 \\
\hline Antibiotic management in pneumonia & 58.0 \\
\hline Management of anaerobic bacteria infection & 38.3 \\
\hline Prevention of catheter-associated UTI & 69.1 \\
\hline Important factors for IV to oral antibiotic switch & 68.7 \\
\hline
\end{tabular}

MRSA: methicillin-resistant Staphylococcus aureus; ESBL: extended-spectrum beta-lactamase; IV: intravenous; UTI: urinary tract infectioin 
The internet was the least utilized among our respondents, contrasting the results of other studies. ${ }^{16,18,20}$ It may not be widely used due to limited internet accessibility in certain hospital areas. Upgrading this infrastructure will help the respondents to get quick access to reliable information in the future.

\section{Perception}

Our respondents unanimously agreed that antibiotics are overused nationally, consistent with the findings from a previous study in Malaysia. ${ }^{18}$ Many studies also concurred that antibiotic overuse and resistance is a significant problem nationwide. ${ }^{7,8,21-28}$

In our study, HO were significantly less confident in prescribing antibiotic optimally compared to $\mathrm{MO}$ and SC. This was not consistent with the study conducted by Leong et al., who reported that there is no statistically significant difference between junior and senior doctors' confidence levels in antibiotic prescribing. ${ }^{18}$ This could be due to the larger sample size in our study. $\mathrm{HO}$ significantly agreed more than MO that they would like more education on antibiotics,

Table IV. Demographic characteristics association with antibiotic knowledge among respondents

\begin{tabular}{|c|c|c|c|}
\hline Demographic Characteristics & & Mean Score \pm SD & p-value \\
\hline \multirow[t]{2}{*}{ Gender } & Male & $5.79 \pm 2.03$ & $p=0.485^{a}$ \\
\hline & Female & $5.97 \pm 1.82$ & \\
\hline \multirow[t]{6}{*}{ Number of years of practice } & Less than 1 year & $4.96 \pm 1.47$ & $p<0.001^{b}$ \\
\hline & 1 to 5 years & $5.70 \pm 1.88$ & \\
\hline & 6 to 10 years & $6.41 \pm 2.18$ & \\
\hline & 11 to 15 years & $7.56 \pm 0.89$ & \\
\hline & 16 to 20 years & $7.15 \pm 1.63$ & \\
\hline & 21 years or more & $7.50 \pm 2.38$ & \\
\hline \multirow[t]{2}{*}{ Local or Overseas Graduates } & Local & $5.97 \pm 1.93$ & $p=0.344^{a}$ \\
\hline & Overseas & $5.71 \pm 1.92$ & \\
\hline \multirow[t]{11}{*}{ Primary Work Area } & Cardiology & $7.00 \pm 1.00$ & $p=0.079^{b}$ \\
\hline & Critical Care & $5.93 \pm 1.72$ & \\
\hline & Endocrinology & $7.50 \pm 2.12$ & \\
\hline & General Medicine & $6.46 \pm 1.86$ & \\
\hline & Infectious Disease & $6.75 \pm 2.50$ & \\
\hline & Nephrology & $6.00 \pm 1.41$ & \\
\hline & Neurology & $6.50 \pm 2.12$ & \\
\hline & Orthopaedics & $5.68 \pm 1.99$ & \\
\hline & Respiratory & $7.25 \pm 2.06$ & \\
\hline & Surgical & $4.33 \pm 2.77$ & \\
\hline & Others & $6.31 \pm 1.95$ & \\
\hline \multirow[t]{3}{*}{ Position } & House Officer & $5.18 \pm 1.53$ & $p<0.001^{b}$ \\
\hline & Medical Officer & $5.67 \pm 1.95$ & \\
\hline & Specialist/Consultant & $7.57 \pm 1.31$ & \\
\hline
\end{tabular}

SD, standard deviation.

Note: alndependent t-test

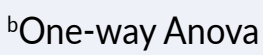


most likely due to the lower level of confidence. In two studies carried out in US and Egypt, majority of the respondents agreed that they would like more education on antibiotics. ${ }^{8,28} \mathrm{~A}$ study in Spain also demonstrated that their resident doctors considered their training regarding antibiotics insufficient. ${ }^{26}$

Our study found that $\mathrm{MO}$ had significantly higher agreement level compared to SC that antibiotic management programmes were an obstacle to good patient care. In local government hospitals, certain selected antibiotics need pre-approval by SC before $\mathrm{HO}$ or $\mathrm{MO}$ are allowed to prescribe. In order not to jeopardize patient care, $\mathrm{MO}$ need to contact the SC via phone consultation. Therefore, MO could have perceived these programmes as being restrictive and time consuming.

This result was consistent with a study done in France and United Kingdom whereby junior doctors favoured more educative interventions such as ensuring the availability of guidelines, educational sessions, and microbiology and ID advice rather than restriction of antibiotics. ${ }^{29}$ Another study done in Philadelphia showed that there is a significant level of frustration among the respondents towards their prior-authorization system due to the time required to call for approval and also the perceived delay in patients receiving antibiotics. ${ }^{27}$ However, this control of antibiotic prescribing is necessary to ensure the appropriate use of the selected broad-spectrum antibiotics as it is proven that the most effective method to control antibiotic resistance are programmes restricting the use of antibiotics and computer-based order forms. ${ }^{30}$ Weaknesses of current antibiotic management programmes should be studied so that areas of improvement can be identified. Meanwhile, continuous education on the importance of current antibiotic management programmes to avoid the development of multidrug-resistant organisms is necessary so that all stake holders understand the rationale behind these programmes.

Our respondents agreed that institutionally developed guidelines for antibiotic treatment would be more useful than national antibiotic guidelines, as observed in other studies.,18,28 Institutionally developed guidelines will take into account the local antibiogram and institutional drug formularies and therefore suit the daily practices at each institution.

\section{Knowledge}

There was no significant difference in knowledge score among different primary work areas. Yetit is interesting to note that the lowest mean score garnered by the surgical department echoes the findings of Srinivasan et al. in 2004. ${ }^{23}$ The question assessing the knowledge of antibiotic usage in surgical prophylaxis scored the lowest percentage of correct answers (21\%) and a previous study found that antibiotic prophylaxis were commonly misused, especially due to inappropriate choice and duration of administration. ${ }^{24}$ Therefore, education on prophylactic antibiotic usage should be emphasized.

Table V. General linear model analysis for demographic characteristics association with antibiotic knowledge among respondents

Demographic Characteristics Adjusted Mean Score \pm SE p-value*

\begin{tabular}{llll}
\hline Position & House Officer & $5.21 \pm 0.30$ & \\
\cline { 2 - 3 } & Medical Officer & $5.68 \pm 0.16$ & $p<0.001^{\text {a }}$ \\
\cline { 2 - 3 } & Specialist/Consultant & $7.58 \pm 0.31$ & \\
\hline
\end{tabular}

SE, standard error.

Note: "Multi-factorial ANOVA test. Main effects of age and number of years of practice were not statistically significant.

apost-hoc Bonferroni's procedures shown significant difference between $\mathrm{HO}$ and $\mathrm{SC}(\mathrm{p}<0.001)$ and $\mathrm{MO}$ and SC $(p<0.001)$. 
The mean scores between the respondents with less years of practice and respondents with more years of practice showed a significant difference, $p<0.001$, corresponding to better antibiotic knowledge due to more exposure and experience. Our result also showed that $\mathrm{SC}$ scored significantly higher than $\mathrm{HO}$ and $\mathrm{MO}$. This was also observed in facility in China where chief doctors were found to possess significantly higher mean knowledge scores compared to other doctors. ${ }^{22}$

A potential limitation in our study includes that our questionnaires were self-reported by the respondents. Social desirability bias was possible as the respondents might provide socially desirable answers instead of actual practices or perceptions. Apart from that, the questionnaires could not be immediately collected back due to time constraints. Hence, the respondents might have discussed among themselves or even searched related references for answers.

\section{Conclusion}

As SC have better knowledge of antibiotic usage, current antibiotic management programmes in Malaysian government that are led by SC need to be continued to ensure judicious use of antibiotics. This study has identified areas worth emphasizing such as confidence in antibiotic prescribing, perception of antibiotic management programmes as an obstacle and also knowledge of antibiotic use in surgical prophylaxis. More targeted antimicrobial education strategy based on the findings of this study should be carried out to improve the practice, perception and knowledge of doctors on antibiotic use and resistance.

\section{Acknowledgements}

We thank the Director General of Health Malaysia for his permission to publish this article, Dr Lilian Abbo for allowing us to use the questionnaire of her team, and the data collectors for assisting with the survey distribution and collection. We would also like to extend our appreciation to all the people that have directly or indirectly helped throughout the process of this study.

\section{Conflict of Interest}

The authors declared no potential conflicts of interest with respect to the research, authorship, and/or publication of this article.

\section{Source of Funding}

The research did not receive any specific grant from funding agencies in the public, commercial, or notfor-profit sectors.

\section{References}

1. Istúriz RE, Carbon C. Antibiotic use in developing countries. Infect Control Hosp Epidemiol 2000; 21(6): 394-397.

2. Sharma M, Eriksson B, Marrone G, Dhaneria S, Lundborg CS. Antibiotic prescribing in two private sector hospitals; one teaching and one non-teaching: a cross-sectional study in Ujjain, India. BMC Infect Dis 2012; 12: 155.

3. National surveillance of antibiotic resistance report ministry of health, Malaysia 2011. https://www.imr.gov.my/images/ uploads/NSAR Archives/Summary of antibiotic resistance 2011.pdf (Accessed 10 April 2019).

4. Okeke IN. Poverty and root causes of resistance in developing countries. In: Antimicrobial Resistance in Developing Countries. New York, NY: Springer New York 2010; 27-35.

5. Protocol on Antimicrobial Stewardship Program in Healthcare Facilities. Malaysia: Ministry of Health Malaysia; 2014.

6. Kheder SI. Physicians' knowledge and perception of antimicrobial resistance: A survey in Khartoum State hospital settings. British J Pharm Res Int 2013; 3(3): 347-362. www. sciencedomain.org (2013, accessed 10 April 2019).

7. Abbo L, Sinkowitz-Cochran R, Smith L, et al. Faculty and resident physicians' attitudes, perceptions, and knowledge about antimicrobial use and resistance. Infect Control Hosp Epidemiol 2011; 32(7): 714-718.

8. Venugopalan V, Trustman N, Manning N, Hashem N, Berkowitz L, Hidayat L. Administration of a survey to evaluate the attitudes of house staff physicians towards antimicrobial resistance and the antimicrobial stewardship programme at a community teaching hospital. J Glob Antimicrob Resist 2016; 4: 21-27.

9. Fine JM, Fine MJ, Galusha D, et al. Patient and hospital characteristics associated with recommended processes of care for elderly patients hospitalized with pneumonia: Results from the Medicare Quality Indicator System Pneumonia Module. Arch Intern Med 2002; 162(7): 827-833.

10. Baadani AM, Baig K, Alfahad WA, Aldalbahi S, Omrani AS. Physicians' knowledge, perceptions, and attitudes toward antimicrobial prescribing in Riyadh, Saudi Arabia. Saudi Med J 2015; 36(5): 613-619 DOI: 10.15537/smj.2015.5.11726. https://doi.org/10.15537/smj.2015.5.11726

11. MOF. National Budget 2019. Minist Financ Malaysia 2018; 79.

12. Pharmaceutical Service Division M. Malaysian Statisctics on Medicines (MSOM) 2011-2014. Minist Heal 2017; 1-254.

13. Sunenshine RH, Liedtke LA, Jernigan DB, Strausbaugh LJ. Role of infectious diseases consultants in management of antimicrobial use in hospitals. Clin Infect Dis 2004; 38(7): 934-938.

14. Al-Tawfiq JA. The pattern and impact of infectious diseases consultation on antimicrobial prescription. J Glob Infect Dis 2013; 5(2): 45-48. 
15. Honda H, Krauss MJ, Jones JC, Olsen MA, Warren DK. The value of infectious diseases consultation in Staphylococcus aureus bacteremia. Am J Med 2010; 123(7): 631-637.

16. Bronzwaer SLAM, Cars O, Buchholz U, et al. A European study on the relationship between antimicrobial use and antimicrobial resistance. Emerg Infect Dis 2002; 8(3): 278282.

17. Kramer MS, Naimark LE, Roberts-Bräuer R, McDougall A, Leduc DG. Risks and benefits of paracetamol antipyresis in young children with fever of presumed viral origin. Lancet 1991; 337(8741): 591-594.

18. Leong TW, Rahmah S, Syahireen N, Zuraidah A. Knowledge, attitude and practice of antibiotics prescribing among medical officers of public health care facilities in the state of Kedah, Malaysia. Med J Malaysia 2015; 70(5): 307-311.

19. Avorn J, Chen M, Hartley R. Scientific versus commercial sources of influence on the prescribing behavior of physicians. Am J Med 1982; 73(1): 4-8.

20. García C, Llamocca LP, García K, et al. Knowledge, attitudes and practice survey about antimicrobial resistance and prescribing among physicians in a hospital setting in Lima, Peru. BMC Clinical Pharmacol 2011; 11: 18. DOI: 10.1186/1472-6904-11-18. https://doi.org/10.1186/14726904-11-18

21. Thriemer K, Katuala Y, Batoko B, et al. Antibiotic prescribing in DR Congo: A knowledge, attitude and practice survey among medical doctors and students. PLoS One 2013; 8(2): e55495.

22. Bai Y, Wang S, Yin X, Bai J, Gong Y, Lu Z. Factors associated with doctors' knowledge on antibiotic use in China. Sci Rep 2016; 6: 23429. DOI: 10.1038/srep23429. https://doi. org/10.1038/srep23429
23. Srinivasan A, Song X, Richards A, Sinkowitz-Cochran R, Cardo $D$, Rand C. A survey of knowledge, attitudes, and beliefs of house staff physicians from various specialties concerning antimicrobial use and resistance. Arch Intern Med 2004; 164: 1451-1456. DOI: 10.1001/archinte.164.13.1451. https:// doi.org/10.1001/archinte.164.13.1451

24. Ng RS, Chong CP. Surgeons' adherence to guidelines for surgical antimicrobial prophylaxis - a review. Australas Med J 2012; 5(10): 534-540.

25. Wester CW, Durairaj L, Evans AT, N Schwartz DN, Husain $\mathrm{S}$, Martinez E. Antibiotic resistance: A survey of physician perceptions. Arch Intern Med 2002; 162(19): 2210-2216.

26. Navarro-San Francisco C, Del Toro MD, Cobo J, et al. Knowledge and perceptions of junior and senior Spanish resident doctors about antibiotic use and resistance: Results of a multicenter survey. Enferm Infecc Microbiol Clin 2013; 31(4): 199-204.

27. Seemungal IA, Bruno CJ. Attitudes of housestaff toward a prior-authorization-based antibiotic stewardship program. Infect Control Hosp Epidemiol 2012; 33(4): 429-431.

28. Fathi I, Sameh O, Abu-Ollo M, et al. Knowledge, attitudes, and beliefs regarding antimicrobial therapy and resistance among physicians in Alexandria University teaching hospitals and the associated prescription habits. Microb Drug Resist 2017; 23(1): 71-78.

29. Pulcini C, Williams F, Molinari N, Davey P, Nathwani D. Junior doctors' knowledge and perceptions of antibiotic resistance and prescribing: A survey in France and Scotland. Clin Microbiol Infect 2011; 17(1): 80-87. https://doi.org/10.1111/ j.1469-0691.2010.03179.x

30. Weinstein RA. Controlling antimicrobial resistance in hospitals: Infection control and use of antibiotics. Emerg Infect Dis. 2001; 7(2): 188-192. 